\title{
Vibrational chiral spectroscopy with femtosecond laser pulses
}

\author{
J. Helbing and M. Bonmarin \\ Universität Zürich, Physikalisch-Chemisches Institut, Winterthurerstrasse 190, CH-8057 Zürich, Switzerland
}

\begin{abstract}
Vibrational circular dichroism and optical rotary dispersion spectra can provide detailed information about molecular structure and the conformation of biomolecules. Their artefact-free recording with high time resolution is a current experimental challenge. We outline recent progress.
\end{abstract}

\section{Introduction}

Vibrational transitions can provide direct access to chemical structure, because they are often localized in different parts of a molecule with well-defined orientations of the transition dipole moments. This is exploited in anisotropy measurements in non-linear spectroscopy. A vibrational band shifts in frequency when another one is excited by a pump-laser pulse, either due to direct coupling of the two vibrations or by heating of the molecule. The dependence of the signal on the orientation of the probe laser polarization is directly related to the angle between the transition dipole moments[1].

For chiral molecules vibrational circular dichroism (VCD) and vibrational optical rotary dispersion (VORD) offer an additional, powerful probe. They can be understood as a measure of the deviation of charge motion during a vibration from that of a linear dipole. Even spectrally unresolved or delocalized modes can thus serve as sensitive indicators of molecular structure, in particular in combination with reliable quantum chemistry calculations[2]. The goal of our current research is to record changes in vibrational chiral spectra in the course of a photo-induced reaction with ultrafast time resolution.

\section{Measurement Principle}

Since vibrational circular dichroism and circular birefringence are very tiny $\left(10^{-3}-10^{-5}\right)$ compared to absorption or linear dichroism of an ensemble of molecules in solution, a conventional polarimetric characterization with mid-infrared light cannot yield chiral vibrational spectra with sufficient precision. Rather than probing the full Mueller matrix of the sample using a set of different polarizations, time-resolved VCD measurements therefore require mid-IR light pulses with a pure, well-defined state of polarization. As samples are usually non-depolarizing, this implies that Jones matrices are sufficient for the description of the experiment.

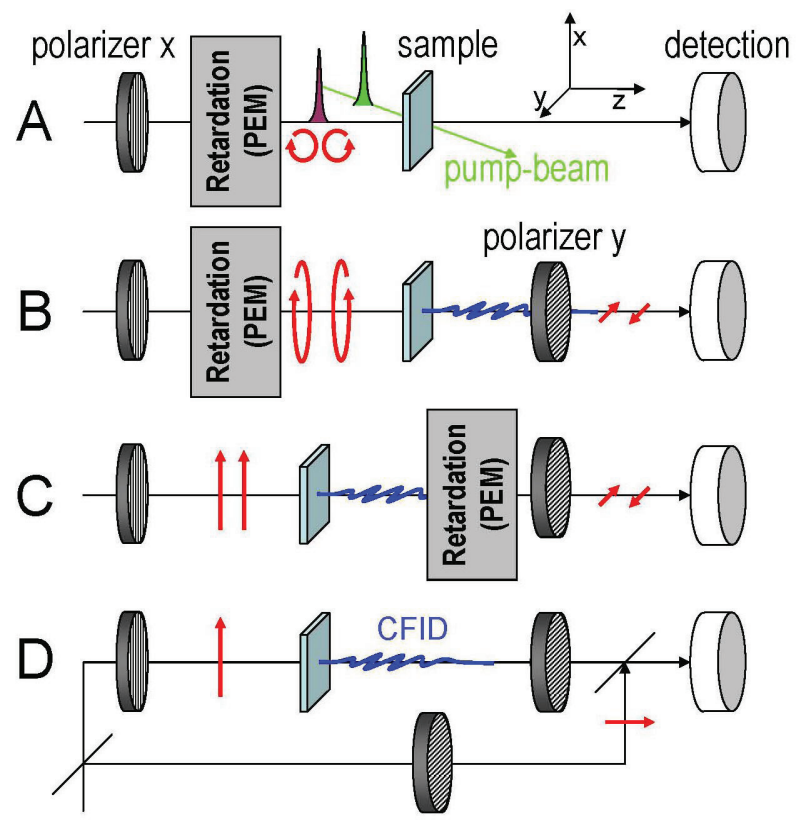

Fig. 1. Principle of time-resolved VCD measuements. A Probe pulse polarizations are alternatingly left and right handed circular (red arrows). They experience different transmission changes after a chiral sample is excitated by a visible pump beam (green). B-D: A second polarizer in the y-direction blocks the $\mathrm{x}$-component of the probe beam and only transmits the $\mathrm{y}$ polarized chiral free induction decay (CFID, blue). The probe beam, and thus the CFID, can be much more intense without saturating the detector. Y-polarized reference fields (with opposite sign for consecutive probe pulses, small red arrows) are created either before the sample (elliptical light, B), behind the sample $(C)$ or guided arround the sample (D). They interfere with the CFID to produce VCD or VORD signals and their pump-induce changes.

This is an Open Access article distributed under the terms of the Creative Commons Attribution-Noncommercial License 3.0, which permits unrestricted use, distribution, and reproduction in any noncommercial medium, provided the original work is properly cited. 
The Jones matrix for a sample of isotropically oriented molecules with weak (vibrational) circular dichroism describes the fact that an initially x-polarized electric field accquires a weak y-polarized component with a $\pi / 2$ phase shift (and vice versa for $y$-polarized incident light):

$$
\begin{aligned}
& E_{\text {in }, x}=\left(\begin{array}{l}
1 \\
0
\end{array}\right), \quad E_{\text {out }}=e^{-\kappa / 2}\left(\begin{array}{c}
1 \\
i V C D-V O R D
\end{array}\right) \\
& E_{\text {in }, y}=\left(\begin{array}{l}
0 \\
1
\end{array}\right), \quad E_{\text {out }}=e^{-\kappa / 2}\left(\begin{array}{c}
-i V C D+V O R D \\
1
\end{array}\right)
\end{aligned}
$$

This new field component is the chiral free induction decay (CFID), which is too weak to be detected unless it is made to interfere with a much stronger reference field. In the case of elliptical polarization,

$$
E_{\text {in }}=\left(\begin{array}{c}
1 \\
\pm i \delta
\end{array}\right), \quad \delta \leq 1
$$

the y-polarized component of the light acts as a reference for the CFID generated by the incident $\mathrm{x}$-polarized component. Indeed, a square-law detector placed behind a polarizer oriented in the y-direction measures

$\left|E_{\text {out }, y}^{ \pm}\right|^{2}=e^{-\kappa}|1 \pm \delta V C D|^{2}$,

and the log-ratio of the signal recorded with left $(+$ sign $)$ and right handed light (- sign) is

$$
\Delta A_{V C D}=\log \frac{\left|E_{\text {out }, y}^{+}\right|^{2}}{\left|E_{\text {out }, y}^{-}\right|^{2}} \approx \frac{4}{\delta \log (10)} V C D \text {. }
$$

The measured VCD signal can thus be strongly enhanced, when highly elliptical $(\delta<<1)$ instead of circular polarized light $(\delta=1)$ is used. However, this comes at the price of an additional polarizer behind the sample, which strongly enhances the sensitivity to linear dichroism and birefringence background signals[3].

It can be seen from equation (1) that to lowest order the $y$-polarized reference field is not affected by the optical activity of the sample. As a result it may be generated only after the beam has passed the sample (Fig.1C). The reference field can even be a replica of the incident field that is guided around the sample cell[4] (Fig. 1D). When the phase difference between the x-polarized incident field and the $y$-polarized reference is turned from $/ \pi \pm 2$ to 0 or $\pi$, it interferes with the in-phase component of the CFID and optical rotation (VORD) instead of circular dichroism is detected[5].

\section{Experiment}

A train of alternating left and right handed mid-infrared laser pulses is created by synchronizing a $1 \mathrm{kHz}$ amplified femtosecond laser system to the intrinsic 50 $\mathrm{kHz}$ frequency of a photoelastic modulator that serves as a variable retarder. The triggers to the laser system are delayed in such a way that the modulator acts as a waveplate with opposite sign for consecutive pulses. Careful control of the exact modulator retardance at the moment a pulse is passing also allows us to compensate linear birefringence of the sample cell and the modulator itself. Almost perfectly symmetric left and right handed pulses can thus be generated, i.e. pulses with identical xcomponents and exactly opposite y-components[6].

The first successful transient VCD experiment was carried out using the experimental configuration in Fig. 1A. A transition metal complex was excited by visible light pulses, and the subsequent change in circular dichroism in the $\mathrm{C}-\mathrm{H}$ stretch region was detected by alternating left- and right handed circular polarized midIR probe pulses with 5 picosecond time resolution[7] (see Fig. 2). In principle, the time resolution of a pump-probe experiment is only limited by the duration of the laser pulses, which is typically 100 fs in our case. However, in the experimental configuration of Fig. 1 the mid-IR probe pulses need to be spectrally narrowed in a monochromator before reaching the sample in order to eliminate all polarization-sensitive optics between sample and detector.

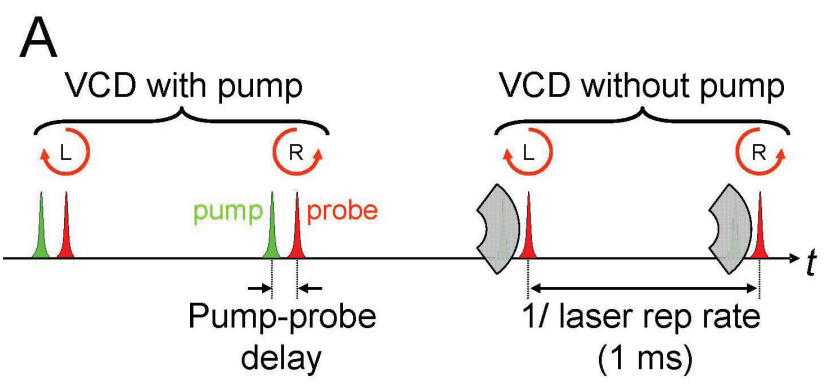

B

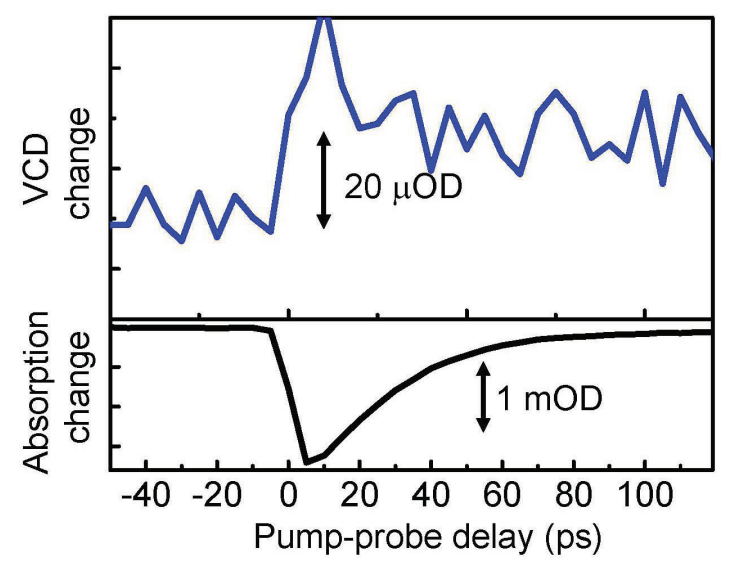

Fig. 2. A Time sequence of pulses in transient VCD spectroscopy. The transient VCD signal is the difference of the VCD measured with and without the pump pulse, which is periodically blocked by a chopper. B VCD (top) and absorption (bttom) changes in the $\mathrm{C}-\mathrm{H}$ stretch region after excitation of the (-)sparteine Cobalt complex $\mathrm{Co}(\mathrm{sp}) \mathrm{Cl}_{2}$ by visible light.

Frequency dispersion behind the additional polarizer is possible for the experimental configurations shown in Fig.1B-D. Not only does this afford femtosecond time resolution but it can also significantly reduce the measurement time when an array detector is used to simultaneously record data over a $100-200 \mathrm{~cm}^{-1}$ frequency range, the typical bandwidth of mid-IR femtosecond laser pulses.

Configurations $1 \mathrm{~B}$ and $1 \mathrm{C}$ have been used with success to record transient electronic $\mathrm{CD}$ and ORD data[8,9] (although UV fs-pulses do not have sufficient bandwidth 
to record spectra), while approach $1 \mathrm{D}$ is only feasible in the mid-IR, as phase stability between the two beam paths is required. Benefitting from the enhancement factor in equation (4) static vibrational $\mathrm{CD}$ and ORD spectra, which are 1-2 orders of magnitude smaller than electronic signals, could recently be recorded using configurations 1B-1D [4,5]. Fig 3 shows VORD spectra of limonene, recently recorded in our laboratory using broad band detection on a 32 pixel MCT detector array [10].
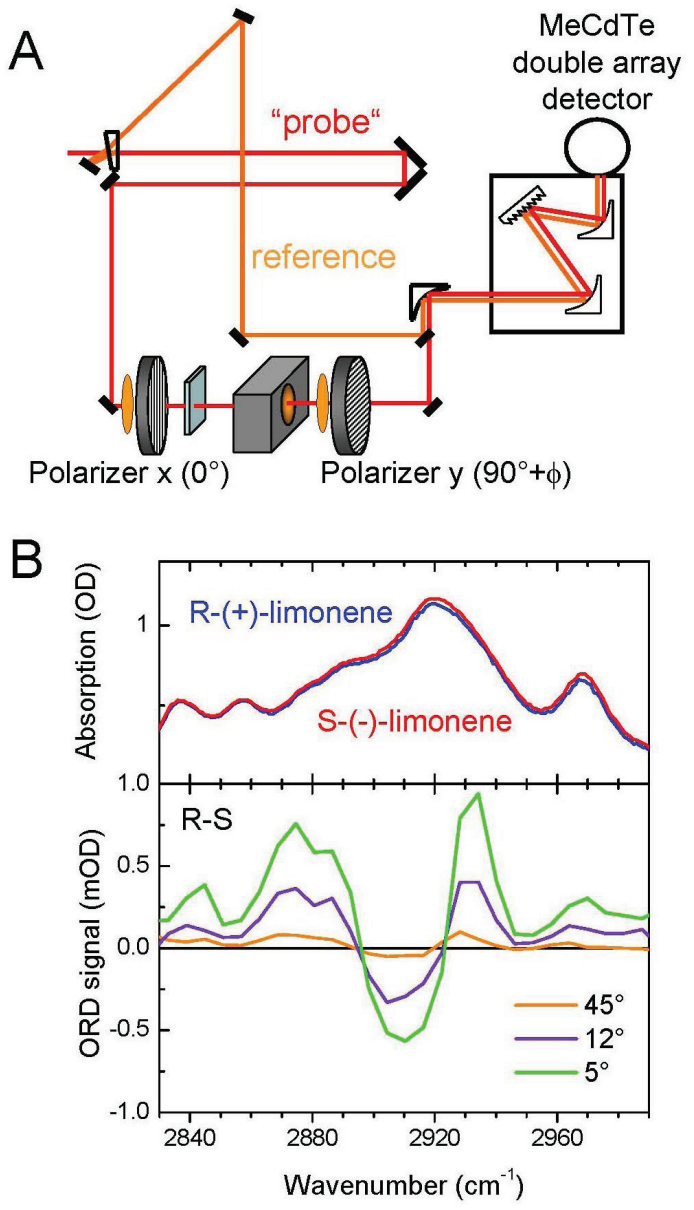

Fig. 3. A Setup for the "single shot" recording of vibrational ORD spectra. The broad-band mid-IR laser beam is split into probe and reference, which are simultaneously recorded on a $2 \times 32$ pixel double array MCT detector. The PEM changes the phase of the y-polarized CFID alternatingly by 0 or $\pi$ with respect to the incident x-polarized laser field. Depending on the detuning $\phi$ of the second polarizer from horizontal, different portions of the incident laser field leak to the detector and act as a reference field. B Absorption and VORD spectra of limonene. Recording time $60 \mathrm{sec}$ for each enantiomer for three different analyser orientations $\phi$.

The high signal levels and short recording times required for these steady state measurements are very promising for the future recording of transient chiral vibrational spectra.

\section{Conclusion}

The vibrational circular dichroism and optical rotation of molecules in solution is very small and detecting its changes during a photoreaction is a challenge in current infrared laser spectroscopy. Concepts borrowed from ellipsometry, combined with a careful control of femtosecond mid-IR pulse polarization have already produced significant signal to noise enhancement in static laser-based chiral measurements. The decisive step toward transient experiments is now the supression of artifact signals due to pump-induced linear birefringence and phase changes.

\section{References}

1. J. Bredenbeck, J. Helbing, and P. Hamm, J. Chem. Phys. 121, 5943 (2004).

2. T. B. Freedman, X. Cao, R. K. Dukor, and L. A. Nafie, Chirality 15, 743 (2003).

3. X. Xie and J. D. Simon, J. Opt. Soc. Am. B 7, 1673 (1990).

4. H. Rhee, Y.-G. June, Z. H. Kim, S.-J. Jeon, and M. Cho, J. Opt. Soc. Am. B 26, 1008 (2009).

5. J. Helbing and M. Bonmarin, J. Chem. Phys. 131, 174507 (2009).

6. M. Bonmarin and J. Helbing, Chirality, early view (2009).

7. M. Bonmarin and J. Helbing, Opt. Lett. 33, 2086 (2008).

8. J. W. Lewis, R. A. Goldbeck, D. S. Kliger, X. Xie, R. C. Dunn, and J. D. Simon, J. Phys. Chem. 96, 5243 (1992).

9. C. Niezborala and F. Hache, J. Opt. Soc. Am. B 23, 2418 (2006).

10. M.Bonmarin, J. Helbing, in preparation. 